\title{
Religion rationnelle et éducation selon Robert Owen
}

Valorisation utopique de l'héritage des Lumières dans l'Écosse du XIX siècle

\section{Claire Puglisi-Kaczmarek}

\section{OpenEdition}

Journals

\section{Édition électronique}

URL : http://journals.openedition.org/etudesecossaises/65

DOI : 10.4000/etudesecossaises. 65

ISSN : 1969-6337

Éditeur

UGA Éditions/Université Grenoble Alpes

\section{Édition imprimée}

Date de publication : 30 janvier 2008

Pagination : 31-49

ISBN : 978-2-84310-110-6

ISSN : $1240-1439$

Référence électronique

Claire Puglisi-Kaczmarek, «Religion rationnelle et éducation selon Robert Owen », Études écossaises [En ligne], 11 | 2008, mis en ligne le 30 janvier 2009, consulté le 08 septembre 2020. URL : http:// journals.openedition.org/etudesecossaises/65; DOI : https://doi.org/10.4000/etudesecossaises.65

Ce document a été généré automatiquement le 8 septembre 2020

(c) Études écossaises 


\title{
Religion rationnelle et éducation selon Robert Owen
}

\author{
Valorisation utopique de l'héritage des Lumières dans l'Écosse du XIX ${ }^{\mathrm{e}}$ \\ siècle*
}

Claire Puglisi-Kaczmarek

1 Réformateur social et éducateur gallois, Robert Owen offre une nouvelle vision de la société tant sur le plan social que du point de vue moral. En effet, il tenta de mettre en place une révolution morale de l'Homme de manière pacifique alors que l'Écosse du début $\mathrm{du} \mathrm{XIX}^{\mathrm{e}}$ siècle vivait des bouleversements économiques et sociaux sans précédents. Empreint d'un esprit encyclopédique, à la manière de ses contemporains, il fit feu de tout bois pour réaliser ses idéaux. Il en résulte une œuvre dense dont la richesse, tant théorique que pratique, doit beaucoup aux apports d'autres penseurs. Aussi, de nombreux aspects de son œuvre théorique et pratique mériteraient une analyse, mais nous allons borner l'étude du noble et ambitieux projet du socialiste utopique à l'analyse de la "Religion rationnelle » à New Lanark. Ainsi nommée par Owen lui-même, la Nouvelle Religion devait remplacer le presbytérianisme existant et autres dogmes religieux pour ensuite procéder à une réforme sociétale. Pour ce faire, il choisit d'expérimenter ses idéaux dans la filature écossaise de New Lanark, qui devint un modèle de l'organisation industrielle, en cristallisant la pensée owéniste. Témoin des conditions de vie des classes laborieuses, Owen ne peut rester passif face au drame humain qui se déroule sous ses yeux. Dès lors, il place l'éducation au centre de ses prérogatives. C'est elle qui constitue le pilier central de son entreprise sociale et qui mérite une attention particulière. L'enjeu est de taille car il s'agit de réformer le système d'instruction de la manufacture au cœur de la Révolution industrielle. Les premières victimes, car les plus faibles, sont les enfants qui sont soumis à des conditions de travail inhumaines. Au début du XIX ${ }^{\mathrm{e}}$ siècle, le travail des enfants dans les usines commence à faire l'objet d'une critique sévère. Les effets corrosifs de la Révolution industrielle provoquent une prise de conscience chez de nombreux philanthropes et économistes tant et si bien que l'état se voit contraint de légiférer sur le travail des enfants à l'usine par le biais du Health and Morals of Apprentices Act en 1802 
et des Factory Acts de 1819 et de 1833. Chacun, avec des convictions différentes, tente de trouver un remède à ces maux. Robert Owen se distingue de ses contemporains par l'aspect laïc de son entreprise et surtout par sa connaissance pointue du monde industriel et ouvrier. C'est en ceci qu'il est novateur.

Dans un premier temps, il conviendra d'étudier les critiques qu'il fait de la société industrielle et religieuse du xix ${ }^{e}$ siècle. À partir de cette critique qui tourne vite à la diatribe, il sera utile de montrer comment il parvient à créer une religion rationnelle à la fois proche de la philosophie des Lumières et malgré tout étonnamment influencée par la tradition judéo-chrétienne. Enfin, il conviendra d'analyser la mise en application de ses idées d'éducation à New Lanark.

\section{Une critique de la société industrielle et religieuse}

Robert Owen naquit à Newtown au Pays de Galles en 1771. Il grandit dans un milieu modeste et alla à l'école jusqu'à l'âge de neuf ans. Plus tard, il fit ses premières armes d'apprenti chez un drapier de Stamford dans le Lincolnshire puis à Londres pour finalement parfaire sa formation dans une grande et prestigieuse filature de coton à Manchester, ville en pleine mutation industrielle. Riche de ces expériences, il se mit à son propre compte à l'âge de dix-huit ans. C'est en 1799 qu'il fit l'acquisition de la Chorton Twist Company, la plus grande filature de coton de Grande-Bretagne située à New Lanark dans le Lanarkshire en Écosse. Owen fit de cette immense fabrique, qui accueillait quelque 2000 ouvriers un grand chantier de rénovation sociale au point de l'ériger en modèle. Des centaines de visiteurs, tel Nicolas, le futur tsar de Russie, vinrent prendre connaissance de son œuvre communautaire, certains pour s'en inspirer, d'autres pour l'inscrire dans le champ de l'utopie. En 1825, après avoir tenté en vain de convaincre la Chambre des Communes du bien-fondé de son projet, il se tourna vers l'Amérique du Nord, dans l'Indiana, où il acheta une parcelle de terre pour y fonder la communauté de New Harmony, cette fois accompagné de ses enfants. En 1827, il mit en vente la filature de New Lanark et laissa ses enfants et autres owénistes poursuivre son œuvre en Amérique. Quant à lui, il continua de mener des projets divers et variés en Grande-Bretagne en participant notamment à la création de syndicats : The Great National Consolidated Trade Union (1834) et The Association of All Classes and All Nations (1835). Toutes ces luttes pour l'égalité sociale et l'amélioration des conditions de vie de la société ont fait de Owen le pionnier du système de l'école élémentaire et le père fondateur du socialisme britannique.

Le moteur de ses prérogatives est son opposition aux effets néfastes de la Révolution industrielle et de la religion. C'est d'ailleurs à partir d'une critique de celles-ci qu'il élabore ses projets de réforme sociétale. À l'instar de nombreux penseurs de l'époque, il dénonce les inégalités sociales engendrées par les mutations économiques. Owen, pour sa part, trouve la situation à New Lanark déplorable, constatant avec émoi que même la filature de son beau-père est le théâtre de tous les vices: "The workers and the apprentices were "ignorant and destitute - generally indolent and much addicted to theft, drunkenness, and falsehood" " ». En 1816, Owen se fait le porte-parole des enfants en témoignant devant la commission parlementaire. À cette occasion, il exprime sa vive opposition à l'embauche d'enfants trop jeunes dans les manufactures, toutes industries confondues. Owen se refuse clairement à employer des enfants âgés de moins de dix ans. Son témoignage préfigure les critiques de Karl Marx : 
Because I consider it would be injurious to the children, not beneficial to the proprietors [...] I very soon discovered that although [at Dale's time] those children were extremly well fed, well clothed, well lodged, and very greatly taken care of them when out of the mills, their growth and their minds were materially injured by being employed at those ages within the cotton mills for eleven hours and half per day $[. . .]^{2}$

Sir Robert Peel, lui-même propriétaire d'usines, écoute attentivement le discours de Owen auquel il est sensible. La proposition de loi est finalement votée et entérinée. Par cet événement, Owen se distingue des philanthropes et économistes de son temps, car il parvient, grâce à la force de la loi, à mettre l'une de ses idées d'humaniste en application. En effet, Owen, contrairement aux économistes libéraux, estime que l'intervention de l'état est nécessaire pour réguler le marché. D'autre part, Roland Marx souligne le caractère moderne de cette législation: «[...] cette loi est historiquement la première loi moderne de protection du travail $[. . .]^{3} »$. En vérité, cet événement dévoile le fond de la pensée du philanthrope. Il s'insurge contre la passivité qui trouve son expression sociale dans la théorie du laissez-faire et de la main invisible défendue par Adam Smith dans The Wealth of Nations.

6 Les économistes libéraux et les premiers socialistes, dont Owen, ont des conceptions divergentes de la loi de la nature ${ }^{4}$. Pour les uns, l'ordre naturel du monde et la nature profonde de l'Homme cohabitent harmonieusement, ce qui ne nécessite pas d'intervention extérieure. Pour les autres, et notamment pour Owen, la nature humaine est duelle : l'Homme est à la fois bon et mauvais. À la manière de Rousseau, le Gallois affirme que la nature de l'Homme est tributaire de l'environnement dans lequel il évolue : c'est essentiellement au sein de la Manchester Literary and Philosophical Society que Owen se familiarise avec les idées des philosophes des Lumières. C'est probablement à cette occasion qu'il adopte cette pensée (qui rappelle Montesquieu) selon laquelle les moeurs de l'être humain sont étroitement tributaires de l'environnement dans lequel il évolue : "That man is formed, prior to and from birth, by the circumstances which surround him, animate and inanimate... That if the circumstances are of a compound nature, some inferior and others superior, so will be the character ${ }^{5}$ ».

7 Dans le contexte de l'époque où l'Homme et sa nature sont mis à rude épreuve, les conséquences sociales et morales ne peuvent s'avérer que désastreuses. Ces circonstances, comme les nomme Owen, exercent une pression sur la nature humaine, qui, à la manière de vases communicants, sollicitent la mauvaise nature de l'Homme et le contraignent naturellement à la corruption tant sociale que morale. Il cite à cet égard l'exemple de la loi de l'offre et de la demande qui encourage l'Homme à acheter bon marché et à revendre plus cher, ce qui le place ainsi dans une situation de concurrence avec son prochain et anéantit les vertus nécessaires à une vie en société harmonieuse :

[...]the formation of minds trained to endeavour to buy cheap and sell dear must be therefore abandoned before man can be educated, to become rational in his feelings, thoughts, and actions, or the Rational System of society can be adopted in pratice [...] This is a principle destructive of all sound and pure morality; it is a principle opposed to the cultivation of all the superior qualities of humanity; it places all individuals in covert enmity to their fellows; it makes man an ignorant and selfish being and creates disorder throughout the mind and conduct of all $;$ it is opposed to sincerity, to charity and to kindness [...] (WRO, vol. 3, p. 375) 
C'est la raison pour laquelle il privilégie le système de fabrique au système de marché. D'où son projet communautaire de villages de coopération avec la fabrique en son centre. Il faut, dit-il, tirer des leçons du passé pour construire l'avenir. Sa préférence pour le système traditionnel de fabrique ne le range pas pour autant dans le camp des passéistes. Il pense, en effet, que la mécanisation et donc le progrès scientifique sont un bien pour la société. Il est donc possible d'inverser les tendances à condition de fournir les moyens nécessaires pour favoriser de meilleures «circonstances ». C'est d'ailleurs précisément grâce au progrès technique qui fait de l'Empire une grande puissance que Owen déclare l'humanité prête à vivre une grande révolution sociale. Progrès technique d'une part, pressions économiques et sociales et détresse humaine d'autre part sont autant de signes à partir desquels Owen avait décrété que le moment opportun était arrivé pour se mettre à pied d'oeuvre. Comme une sonnette d'alarme, le drame humain qui s'opérait sous ses yeux, était un signe évident que la société était prête à vivre un grand changement, une révolution morale.

9 Parmi les socialistes se trouvent des chrétiens qui ont énoncé les mêmes critiques de la société capitaliste naissante. C'est notamment l'un de ses contemporains, Thomas Chalmers (1780-1847), théologien écossais, réformateur social et figure emblématique des mouvements évangéliques qui a tenté par des moyens tant caritatifs que doctrinaires d'enrayer les conséquences désastreuses de la Révolution industrielle. Le démon du capitalisme, pour Owen comme pour Chalmers (qui donne au capitalisme le nom de la divinité Mammon), est l'argent et son pouvoir destructeur :

The God of the world is wealth, individual wealth; the great stimulus to action among men is to obtain it; all the superior or happiness-producing qualities of our nature are held in abeyance, that the wealth-obtaining qualities may have unrestrained action. And yet all this is rank insanity or downright madness. (Ibid., p. 372)

10 Il semble donc qu'il y ait un véritable consensus sur ces questions. Toutefois, bien que Owen et Chalmers semblent combattre la même bête qu'incarne le capitalisme, parfois avec le même arsenal, il n'en reste pas moins qu'un mur se dresse entre eux. En effet, Owen tient la religion personnellement responsable de tous ces maux et c'est précisément en faisant une critique de celle-ci qu'il peut procéder à la réforme de la société.

11 Les premiers rapports de Owen avec la religion vont, semble-t-il, définitivement conditionner son point de vue sur celle-ci. Dans sa jeunesse, Owen avait accès à une bibliothèque. Ses propriétaires, l'un presbytérien et l'autre épiscopalien, possédaient des ouvrages religieux qu'il consultait de temps à autre. À cette même époque, Owen assistait aux services religieux des deux confessions. Il fut alors le témoin de leurs querelles dogmatiques. Fort de ses lectures et de cette expérience, Owen se fit donc une opinion solide sur la religion traditionnelle. Ce serait alors à cette époque que le mot religion aurait pris tout son sens pour lui. Un sentiment anti-religieux marquera définitivement son œuvre, sentiment qui ne fera que grandir lors de ses expériences dans le monde industriel.

12 C'est particulièrement dans ses ouvrages qu'Owen se montre hostile envers la religion, estimant que le monde est géré par celle-ci et que ses effets sont dévastateurs : « [...] the religion of the world is the sole cause now of all the disunion, hatred, uncharitableness, and crime, which pervade the population of the earth [...]» (WRO, vol. 2, p. 181). En tout cas, on peut certainement retenir de son discours que la religion 
serait ainsi la cause première de tous les maux de la société, notamment celle de l'ignorance :

The present system, having been based fron the beginning on ignorance, and hitherto sustained solely by ignorance, should this ignorance be superseded by real knowledge, the whole of the existing system and organization of society would appear so monstruous, contradictory, and absurd, that none, after a short period, could be otherwise than greatly ashamed to remain an advocate for the continuance of such an heterogeneous mass of sin and misery, of gross irrationality, and obstruction to human happiness. (WRO, vol. 3, p. 366)

13 Progressivement, au fil de son discours, on comprend qu'Owen confond religion et superstition. L'ignorance qu'il déplore est continuellement alimentée par les superstitions : «In the progress from ignorance to real knowledge, the millions have discovered the fallacy of the foundation on which all the various superstitions of the world from the beginning have been constructed » (Ibid.). Il faut donc se replonger dans le contexte historique pour constater que c'était bel et bien la voie qu'avait prise la religion, celle du fondamentalisme, à travers les mouvements de Réveils. Owen accuse essentiellement les prédicateurs évangéliques issus de ces mouvements, citant en particulier la Clapham sect et l'évangélique et parlementaire William Wilberforce, qu'il compte parmi les «amiable weakmen »: «The furious bigot [...] who keeps all around him in a continual state of ignorant excitement " (WRO, vol. 2, p. 172). Du nom d'un village situé près de Londres, le mouvement de Clapham regroupe des prédicateurs et hommes politiques influents qui se sont rassemblés afin de combattre l'esclavage. Ce mouvement fut très actif jusqu'en 1833, date qui correspond, en Grande- Bretagne, à l'abolition de l'esclavage, avec la promulgation du Slavery Abolition Act. Certes engagé dans les grandes questions sociales et morales de son temps, le mouvement avait aussi toutes les caractéristiques de l'évangélisme du début du XIX siècle. En 1797, Wilberforce avait publié son premier livre, A Practical View of the Prevailing Religious System of Professed Christians in the Higher and Middle Classes of this Country contrasted with Real Christianity, ouvrage de prosélytisme dans lequel il appelait au réveil du christianisme et à un retour à la moralité d'antan. En raison des événements de la Révolution française, de la sécularisation grandissante et du désengagement progressif de l'État envers la religion, notamment envers les Églises d'État comme la Kirk, les mouvements de Réveils s'étaient renforcés ${ }^{6}$. Leurs méthodes étaient théâtrales et frisaient parfois le ridicule comme en témoigne Robert Burns dans l'un de ses poèmes, Holy Fair. Les philosophes des Lumières comme John Locke et David Hume avaient déjà exprimé leurs vives critiques envers ces enthousiastes ${ }^{7}$. C'est donc dans cette continuité qu'Owen avait jugé ces communautés religieuses. À son tour, il s'insurge contre ces pratiques sectaires qui anéantissent les capacités de réflexion du croyant qui est, depuis son enfance, enfermé dans un carcan doctrinaire : «And as none of this class are ever permitted to investigate the laws of their nature, but are trained from infancy to believe and not to think» (WRO, vol. 2, p. 172). Selon Owen, cette ignorance est fondée sur des superstitions qui n'émanent pas de l'expérience : «[...] superstitions have one and all emanated from inexperienced, and often greatly diseased imaginations of individuals [...]» (WRO, vol. 3, p. 366). Le raisonnement d'Owen consisterait donc à mettre en opposition la Révélation divine et l'expérience. Ainsi, la croyance en des superstitions est en opposition avec les lois de la nature fondées sur l'expérience. Il s'agit, chez Owen, de former des êtres pensants. C'est dans cette optique qu'il entend 
réformer la société : A New Moral World. Pour ce faire, il faut soumettre la connaissance à l'expérience par le truchement de nos sens.

Le penseur ne se contente pas de suggérer ses méthodes à autrui. En effet, il s'applique en premier lieu cette méthode empirique à lui- même lorsqu'il juge la religion en nous fournissant une définition historique de celle-ci, et c'est bien par ce biais qu'il se permet d'incriminer la religion. À en juger par son passé, la religion chrétienne, telle qu'elle est pratiquée depuis des siècles, est incompatible avec la religion naturelle :

Religion [...] is an attempt to force mankind to think against the evidence of their senses and of all facts, that there is merit of the highest order in believing what the priests say their votaries ought to believe, and the deepest of all demerits in not believing these dogmas, which they one and all, whatever may be the name and form which they support, agree to call divine truths [...] (WRO, vol. 2, p. 176)

Mais au fond, lui aussi reconstruit un dogme, car son projet communautaire sous la forme de villages de coopération implique des règles strictes. La preuve en est qu'il arrivait que certains ouvriers quittassent définitivement la filature pour rejoindre la fabrique voisine de Blantyre. En 1816, le propriétaire de Blantyre se fait le porte-parole d'une ouvrière qui s'était lassée du paternalisme de Owen :

She said that there had been a number of new regulations introduced. That they had got a number of dancing- masters, a fidler, a band of music, that there were drills and exercises, and that they were dancing together till they were more fatigued than if they were working. - Did the woman complain that she got no wages for the dancing? - no she did not ${ }^{8}$.

On peut s'interroger si Owen, de son côté, ne souhaitait pas aussi faire de ses ouvriers des enthousiastes, afin de les anesthésier pour mieux mettre en œuvre ses projets. En fin de compte, la quête du bonheur universel d'Owen a tout d'une religion : la Religion Rationnelle. Paradoxalement, l'expression de Karl Marx, «la religion est l'opium du peuple » peut aussi s'appliquer à la religion rationnelle. On peut à présent se demander en quoi consiste cette religion rationnelle qu'il nous propose en lieu et place de la traditionnelle.

\section{Une nouvelle religion : la Religion de la Charité}

17 La nouvelle religion est ce qu'il nomme la Religion de la Charité : «[...] Faith could be of no practical value whatever [...]. now from henceforth Charity presides over the destinies of the world ${ }^{9}$ ». En ceci, Owen n'est pas très original car la charité est l'un des piliers du christianisme. L'Homme est appelé à aider son prochain de manière désintéressée. Le terme charité vient de caritas en latin, donc amour. Dans le Nouveau Testament, on emploie l'expression grecque de Agapé. Il s'agirait donc d'aimer Dieu et ses semblables, et la charité en serait la parfaite expression. Dans la Bible, c'est l'apôtre Paul qui invite le chrétien à faire acte de charité :

Sans la charité, je ne suis rien [...] Sans la charité, cela ne me sert de rien. La charité prend patience, la charité rend service, elle ne jalouse pas, elle ne plastronne pas, elle ne s'enfle pas d'orgueil, elle ne fait rien de laid, elle ne cherche pas son intérêt, elle ne s'irrite pas et n'entretient pas de rancune, elle ne se réjouit pas de l'injustice mais trouve sa joie dans tout, elle excuse tout, elle croit tout, elle espère tout, elle endure tout [...] les trois demeurent : la foi, l'espérance et la charité. Mais la charité est la plus grande ${ }^{10}$. 
Au regard de cette pensée, Owen aurait pu être l'auteur de ces versets sauf en ce qui concerne le dernier car la charité à laquelle le rationaliste fait allusion n'est plus liée à la foi : «[...] the religion of Charity, unconnected with faith, is established for ever. Mental liberty for man is secured, and hereafter he will become a reasonable and consequently a superior being " (EW, vol. 1, p. 229). Le Nouvel Homme édifié par Owen doit retrouver par là même sa « liberté mentale » car il n'aura plus la foi en ce Dieu.

Une fois que l'Homme est libéré de ses chaînes dogmatiques, le privant ainsi de cette foi, source de tous les maux, il n'y a plus aucune obstruction à la manifestation de ce qu'Owen reconnaît comme la charité. Cette vertu innée procède de la bonté. Comme le rationaliste estime qu'il $\mathrm{y}$ a du bon en l'Homme, il considère ainsi qu'il n'est pas condamné à une destinée tragique ${ }^{11}$. Sans cette bonté innée, il n'y a point d'acte charitable. La religion, selon Owen, est un obstacle à la bonté naturelle de l'Homme, contraint d'obéir à un dogme et donc privé de sa liberté. Dans le christianisme, l'Homme vit dans une condition de péché et de culpabilité depuis la Chute. Tentant de racheter ses péchés, l'Homme s'investit dans des oeuvres rédemptrices, souvent caritatives $^{12}$. Owen veut faire de la bonté, et donc de la charité, un acte naturel et spontané qui ne cache aucun dessein eschatologique ni calcul. La vertu de la charité devient donc le maillon qui relie les hommes entre eux : la religion.

On constate, par conséquent, que les tensions entre le croyant et le rationaliste se nouent au coeur de la conception de la nature humaine. Si la nature de l'Homme est fixe selon le chrétien, elle est, pour Owen, sur le point de vivre une révolution à l'aune de la révolution scientifique: «[...] the irresistable progress of nature's great change [...] » (WRO, vol. 3, p. 379). Mais une fois les fondements de la nature redéfinis, il faut, selon Owen, les accepter comme acquis et définitifs :

[...] to establish upon a solid and everlasting foundation, the permanent system of nature, based upon unchanging facts, which never err, or are inconsistent with themselves and all other facts, and which facts lead alone to real knowledge and substantial happiness [...] (Ibid.)

The laws of nature never change for man, or any earthly affairs or proceedings whatever. (WRO, vol. 2, p. 181)

21 Ainsi, si l'on note, à moment donné, que l'heure est venue pour une Révolution morale chez Owen, les deux cosmogonies finissent tout de même par se rejoindre.

Mais ces lois de la nature qui sont fixes trouvent leurs racines en un Dieu de la Nature omnipotent. Owen donne une puissance divine à la Nature, car qui s'y oppose est condamné à la défaite : "nature is against the continuance of the present irrational system ; and they who oppose nature, who never gives up the contest must sooner or later be defeated (WRO, vol. 3, p. 382). Le dessein de Owen est de façonner la moralité des Hommes, naturellement doués de raison, au regard de cette Nature. Pour les chrétiens, l'Homme procède du Créateur et serait fait à son image, Imago Dei. Ainsi, ce consensus révélerait que les natures des deux divinités sont très proches, ce qui supposerait donc que l'interprétation de la nature humaine par les chrétiens et les rationalistes est similaire :

Que l'on comprenne l'Homme comme sortant des mains de Dieu ou bien de la Nature, l'interprétation demeure la même. Thomas More parle de l'homme sortant des mains de son Créateur. Les penseurs de l'époque des Lumières parlent de l'homme sortant des mains de la Nature ${ }^{13}$. Dieu chrétien, est diamétralement opposé à la Nature. Afin de mieux comprendre les 
divergences de point de vue entre le croyant et le rationaliste, il convient de remonter aux origines de la Charité. La source de la religion chrétienne serait, selon Owen, une divinité abjecte et dédaigneuse pour avoir répandu autant de malheurs sur le monde :

If, indeed, religion, as it has been hitherto taught, has emanated from a divinity, it must have been from one possessing the most dire hatred to mankind; one, who well knew how the most effectually to destroy, in the bud, the finest qualities of human nature, and the highest enjoyments of every individual (WRO, vol. 2, p. 192).

Dès lors, le rationaliste détrône cette divinité pour la remplacer par celle de la Vérité. Owen aborde ce qu'il appelle: "The Second coming of Truth» (WRO, vol. 3, p. 405). On ne peut que faire la comparaison avec La Seconde Venue du Christ. Ses sources sont bibliques puisqu'il cite un passage du Nouveau Testament, d'abord pour illustrer la première venue du Christ (Ibid., p. 405-406). La seconde venue de la Vérité est celle préconisée par Owen, comme s'il ré-écrivait le Nouveau Testament. De son vivant, l'Homme va sortir alors de l'ignorance pour acquérir une connaissance, fruit de $L a$ Seconde Venue de la Vérité. Dans une certaine mesure, il semble qu'Owen joue alors un rôle de prophète. En cette qualité, il annonce la fin du Règne de la Foi :

Yes, my friends, in the day and hour when I disclaimed all connexion with the errors and prejudices of the old system - a day to be remembered with joy and gladness henceforward throughout all future ages - the Dominion of Faith ceased; its reign of terror, of disunion, of separation was broken to pieces like a patter's vessel (see Psalms 2:9). The folly and madness of its votaries became instantly conspicuous to the world. (EW, vol. 1, p. 227)

Enfin, le rationaliste se montre pratique dans la mesure où ses initiatives, qui sont censées conduire au bonheur, doivent être mises en application le plus tôt possible. Il envisage, en effet, de fonder un paradis terrestre, "to make the earth a terrestrial paradise » (WRO, vol. 3, p. 381) afin de sortir le peuple de l'ombre, «Egyptian darkness » (La Bible, L'Exode 8:6) (Ibid., p. 380). Cet idéal est commun à d'autres groupes religieux, notamment à la Kirk. Cependant, toutes les confessions protestantes n'ont pas la même conception théologique du bonheur et donc du paradis. En effet, pour les Églises dissidentes, le bonheur n'est pas de notre monde tandis que pour la Kirk, et à travers l'idéal chalmérien, il est possible de construire notre bonheur sur terre. Ces deux conceptions différentes du bonheur, l'une spirituelle, donc relevant de l'eschatologie, et l'autre immédiate, donc temporelle, donnent lieu à des comportements et à des choix politiques et sociaux très différents. La Kirk souhaite coopérer avec l'État pour accélérer la mise en place du bonheur terrestre tandis que les dissidents s'opposent à l'ingérence de l'État dans ses affaires spirituelles. Cette intrusion ne participe pas du bonheur spirituel qui se révélera à la fin des temps. Quant à Owen, il souhaite, au contraire, la coopération de l'État puisqu'il n'hésite pas à transmettre au Parlement ses requêtes et ses travaux. Il invite d'ailleurs à New Lanark des hommes d'influence, du futur Tsar de Russie aux hommes politiques britanniques. Mais Owen est aussi un riche industriel, c'est peut-être la raison pour laquelle il pouvait se permettre d'écorner l'image de la religion et d'attiser, au moins dans les premiers temps, la curiosité des hommes les plus influents de Grande-Bretagne et d'ailleurs. Mais de cette vision du bonheur, découlent aussi des conceptions différentes de l'organisation de la société et en particulier de la notion de travail. Tandis que les Églises, pour des raisons tant théologiques que politiques, souhaitent maintenir la hiérarchie sociale en l'état, comme le suggère, au fond, Thomas Chalmers (qui souhaite éduquer moralement les pauvres), Owen voit dans le progrès scientifique un allié de 
l'émancipation sociale de l'Homme qui est jusqu'alors enfermé dans un carcan tant moral que physique : «mental slavery and physical servitude » (Ibid., p. 379). Nul besoin d'esclaves pour faire le travail puisque les machines remplacent peu à peu l'Homme. Dans la tradition chrétienne, les Églises protestantes et calvinistes ne se sont pas opposées à la hiérarchie sociale existante en raison de leurs conceptions de la doctrine de la prédestination. Jean Calvin développe cette doctrine dans Institution de la religion chrétienne en 1536. Sa thèse présuppose la prédestination à être élu et à recevoir la grâce, thèse qui a pu aussi justifier la condition sociale, pour les pauvres comme pour les riches. La célèbre thèse de Max Weber cherche à démontrer que cette doctrine a contribué au développement de l'esprit capitaliste des chrétiens, le travail et la richesse étant considérés comme une grâce de Dieu, un don et un signe de l'élection divine ${ }^{14}$. C'est bien plus à travers le travail, dont la richesse est la manifestation, qu'à travers l'argent à proprement parler que le calviniste participe au capitalisme. Mais cette position fait encore aujourd'hui l'objet de vifs débats.

$\mathrm{Au}$ sein de la communauté, le travail, pour Owen, devient la seule richesse et c'est par conséquent autour de ce principe qu'il organise toute la société. Mais afin qu'éthique et travail se répondent dans une parfaite harmonie, le rationaliste s'engage à mettre un terme à la hiérarchie sociale. Il souligne en particulier l'égalité dans l'éducation et dans la condition sociale: "To those who understand human nature it is now evident that there can be no peace, no unity, no permanent prosperity and happiness, so long as there shall be inequality of education or condition " (WRO, vol. 3, p. 377-378). Afin de briser le schéma classique, Owen favorise une classification de la société en fonction de l'âge : "The classification of age is the essence of justice to the human race, and will produce universal charity, kindness, peace, and happiness; while the classification of birth and wealth, will, so long as it shall be most unwisely permitted, produce uncharitableness, unkindness, wars and misery » (Ibid., p. 407-408).

La rupture entre le religieux et le rationaliste est ici définitivement consommée. Après avoir fait table rase des pratiques religieuses existantes, Owen souhaite éduquer la population sur des bases saines. C'est par le truchement de ses expérimentations à New Lanark qu'il tente de mettre sur pied un nouveau système d'éducation pour édifier conjointement la Religion de la Charité et le Nouvel Homme. Pour ce faire, il convient d'associer étroitement instruction et éducation dans un environnement industriel.

\section{L'exemple de la filature de New Lanark : un système d'éducation à la croisée du rationalisme et de la tradition knoxienne}

Nous pouvons conclure de ce qui précède que tous les vices de la société (pauvreté, superstitions, crimes et violence) de la première moitié du XIXe siècle sont imputables, selon Owen, à une mauvaise éducation. Dès lors, l'éducation devient un pilier central de la réforme sociétale de Owen :

[...] Man must be educated. But how ? No longer to be a mental and physical slave to imaginary fears; the days of ghost and hobgoblins are passing with the superstitions which gave them birth, and man must have his character henceforward based on demonstrable truth and must be made an intelligent and superior rational being, fitted to create the circumstances to ensure happiness to his offspring, and to live a long life of healthy joyous existence. (Ibid., p. 369) 
Depuis le Moyen-Âge, le clergé catholique avait fait main basse sur l'instruction. Le réformateur John Knox reprit le flambeau et stipula clairement, dans le Livre de Discipline, que l'instruction religieuse devait être prodiguée à tous les enfants de chaque paroisse. La paroisse avec son école en son centre devint la caractéristique du presbytérianisme écossais. De ce projet très ambitieux naquit un mythe selon lequel l'Écosse était l'un des pays les plus lettrés d'Europe. C'est à la fin du dix-huitième siècle que l'instruction connut son âge d'or: la Kirk pouvait alors se vanter d'avoir un pastorat éduqué. Tobias Smollett en fait d'ailleurs la remarque: "Even the Kirk of Scotland, so long reproached with fanaticism and canting, abounds at present with ministers celebrated for their learning, and respectable for their moderation ${ }^{16} »$. Pour renforcer le mythe, il est dit que des enfants modestes, souvent issus d'un milieu rural, avaient été promus à un rang social élevé : le lad o'pairts. Mais au début du XIX ${ }^{\mathrm{e}}$ siècle, la Kirk, dominant encore le paysage de l'instruction, n'était plus la seule Église à prodiguer une instruction, en particulier dans les villes. Il y en avait pour les riches et pour les pauvres. Les riches, d'abord, qui bénéficiaient des prestigieuses Grammar Schools. Et les plus modestes pouvaient s'instruire dans des écoles de charité, parfois par le biais de maîtres d'école itinérants, et surtout grâce aux écoles du dimanche en grande partie dynamisées par les Éveils religieux.

32 Grâce à l'école paroissiale, John Knox était parvenu à instiller les préceptes de la Réforme, à se garantir contre tout retour des prélats, à maintenir une relative paix sociale et surtout à pérenniser l'existence de la Kirk. Owen n'est donc pas le premier réformateur à vouloir faire de l'éducation un principe fondamental de son programme. Thomas Chalmers, considéré comme le troisième réformateur, après le grand réformateur John Knox et le covenantaire Henderson, a tout misé sur l'éducation pour extirper la population de son bourbier moral et social. Son projet demeurait à forte coloration religieuse, dans la continuité de l'oeuvre knoxienne, en prenant soin toutefois d'adapter le système traditionnel aux nouveaux enjeux socio-économiques. Mais son concurrent, Owen, était novateur, offrant à la population ouvrière une alternative à l'instruction religieuse traditionnelle: l'école laïque. L'instruction devenait un enjeu idéologique.

33 Microcosme de toutes les spécificités économiques et sociales de l'Écosse du xIXe siècle, la filature de New Lanark se présentait comme le laboratoire expérimental idéal. Toutes les conditions étaient réunies, car cette filature, archétype de l'industrialisation, était la plus grande manufacture de coton de Grande Bretagne qui était à la pointe du progrès scientifique en matière de machines et qui rapportait à son propriétaire, David Dale, une immense richesse.

David Dale (1739-1806) avait acquis la manufacture de New Lanark en 1785. De nature charitable, il avait mené son affaire avec beaucoup d'humanité. Contrairement à de nombreux industriels, Dale investissait une partie des richesses de la manufacture dans les oeuvres caritatives, notamment dans les écoles et dans les hôpitaux. Philanthrope, le maître de New Lanark devait ses qualités d'altruiste à sa foi. D'abord membre de 
l'Église d'Écosse, la Kirk, il rejoint les rangs de la Relief Church pour ensuite devenir prédicateur dans la confession indépendante des old Scots Independents. De confession presbytérienne donc, Dale laissait à Owen une filature qui avait certes de nombreuses lacunes mais qui avait fait l'objet d'une attention particulière quant à l'organisation de son système d'instruction, avec en particulier une crèche et une école maternelle. Fruit de l'école paroissiale, Dale en avait gardé l'organisation : « Dale's schools in New Lanark retained elements of the parish system, most notably in their emphasis to reading and catechism ${ }^{17}$ ».

Robert Owen succéda à l'ancien propriétaire, David Dale, dont il épousa la fille, Caroline. Une fois propriétaire de la filature, Owen prit rapidement les mesures nécessaires pour réduire le temps de travail des ouvriers, le limitant à dix heures par jour. Il se refusa à embaucher des enfants de moins de dix ans. Enfin, il leur prodigua une instruction gratuite jusqu'à l'âge de 12 ans.

Malgré ces nobles initiatives, les critiques acerbes de Owen contre la religion suscitèrent bientôt le mécontentement du presbytère local de la Kirk. Certes critique envers la religion, Owen n'en demeurait pas moins un homme tolérant. Il tenait compte malgré tout des requêtes de ses ouvriers en employant un ministre du culte. Si nécessaire, il faisait même appel à un pasteur pour les services en gaélique destinés aux ouvriers originaires des Hautes Terres. Il s'offrit même les services du révérend Dr John MacDonald, figure évangélique des Hautes Terres. Owen commençait aussi à adopter les pratiques religieuses sous son propre toit : «By Macnab's account religious observance was the rule and at Owen's own house at Braxfield daily prayers were observed by a large and moral family ${ }^{18}$ ». Le philanthrope partageait la direction de la filature avec trois autres associés Quakers, Allen, Foster et Gibbs. Ces derniers se préoccupaient de la persistance de Owen à affirmer publiquement son opinion anti-religieuse. Il faut comprendre que même si Owen peut apparaître modéré pour le lecteur d'aujourd'hui, ses positions apparaissaient excessives dans une société où la religion avait encore droit de cité et où le clergé tenait le haut du pavé. Même si les trois Quakers résidaient à Londres - ce qui laissait une certaine marge de manoeuvre à Owen, ils venaient régulièrement s'enquérir de la santé morale et spirituelle de la communauté.

Entre quatre cents et cinq cents enfants, en rang militaire et vêtus d'un kilt, chantaient des chansons profanes. Owen avait pris soin de remplacer les leçons de chant liturgique mises en place par son beau-père. Les pasteurs de New Lanark témoignèrent malgré tout des bonnes moeurs de ses habitants ${ }^{19}$. Mais Owen, sous couvert de tolérance, souhaitait réformer les moeurs en douceur. Aussi, commença-t-il à préconiser que soit remplacée la lecture de la Bible par des leçons de géographie. Bientôt, les membres du Presbytère de la Kirk eurent vent de ces réformes et ne tardèrent pas à condamner cette mesure. Dès lors, New Lanark fut montré du doigt comme l'antre de l'athéisme : « It is a system of undisguised Atheism and social corruption ${ }^{20} »$. Les chrétiens vilipendaient ces initiatives. Rien ne pouvait davantage rappeler les trois associés Quakers à leurs devoirs moraux. En bons cléricaux qui se respectent, ils durent intervenir pour rectifier les mesures prises par Owen, en réintroduisant l'instruction religieuse et en supprimant les cours de danse. Owen faisait grise mine, mais il était parvenu à trouver une via média en choisissant de fournir une instruction religieuse qui ne devait en aucun cas refléter une confession particulière. La Bible devait figurer comme un support de lecture parmi d'autres. 

qu'Owen, ouvertement sceptique, ait pris fait et cause pour les sociétés bibliques. En effet, l'école était en partie financée par la British and Foreign Bible School Society, société philanthropique religieuse qu'il admirait particulièrement. En effet, il saluait le travail exemplaire que la société avait accompli. Elle avait su condamner les formes les plus extrêmes de l'évangélisme: "But the belief in, and reverence for, these systems for deranging the faculties, and creating the most vile and violent of human passions, are rapidly decreasing throughout the world ; and that decrease is mightly hastened by the establishment and progress of the British and Foreign Bible Society (WRO, vol. 3, p. 367). On peut tenter de justifier cette contradiction chez le rationaliste de plusieurs façons. D'une part, c'était Robert Dale, son beau-père, qui avait fondé la première société biblique d'Écosse à Glasgow en 1805. L'ensemble des sociétés devait soutenir la British and Foreign Bible Society, la maison mère, fondée en 1804 à Londres. Thomas Chalmers était lui aussi très impliqué dans le développement de ces sociétés. Il en avait d'ailleurs exprimé sa plus vive admiration: "The Christianity of the Bible gains a readier access into the hearts of the ignorant than the Christianity of sermons and systems and human compositions ${ }^{21}$ ». C'est probablement sur ce point que les deux réformateurs se rejoignent. La société à vocation missionnaire symbolisait, dans une certaine mesure, la lutte contre les superstitions et donc contre l'ignorance.

l'obédience. Très admiratif des systèmes d'instruction d'Andrew Bell et de Joseph Lancaster, il se mit à appliquer scrupuleusement leurs méthodes, qui concordaient avec son idéal d'éducation des classes laborieuses ${ }^{22}$. Même si les pédagogues accordaient une place prépondérante à l'instruction religieuse dans leur curriculum, Owen fut d'abord séduit par le fait qu'ils menaient leur entreprise en dehors des circuits de l'Église anglicane, dont ils étaient membres ${ }^{23}$. Ensuite, le principe du monitorat, selon lequel les élèves plus doués aidaient leurs camarades, s'accommodait bien avec les vertus d'entreaide et de bonté qu'il souhaitait inculquer aux enfants. Pour Owen, ces méthodes pédagogiques novatrices étaient plus utiles que l'apprentissage des « trois $\mathrm{R}$ ».

$\mathrm{Au}$ fil du temps, Owen prend de la distance par rapport aux deux pionniers pour suivre une voie qui lui est propre. En effet, l'entraide à l'école est vaine si le maittre d'école n'est pas formé convenablement selon les besoins pratiques de l'enfant. Ainsi, en soulignant cette nécessité, serait-il devenu le pionnier de l'école normale.

Dans ce réajustement, le philanthrope souligne la nécessité d'une instruction pratique et non théorique. Aussi critique-t-il vivement les méthodes traditionnelles de la mémorisation. Selon Owen, la mémorisation n'apprend pas à l'enfant à réfléchir mais à restituer un savoir, souvent sans en comprendre le sens. C'est d'ailleurs ce qu'il reproche aux confessions religieuses qui inculquent le catéchisme. Dans son organisation, les parents devaient jouer un rôle fondamental dans l'éducation de leur progéniture en les encourageant dans leurs études et en supprimant toute forme de punition et de récompense, l'enfant devant tirer lui-même des leçons de ses erreurs. À cet égard, Owen se serait inspiré de l'éducateur John Locke :

Beating, then, and all other sorts of slavish and corporal punishments are not the discipline fit to be used in the education of those we would have wise, good, and ingenuous men [...] On the other side, to flatter children by rewards of things that are pleasant to them is carefully to be avoided ${ }^{24}$. 
d'autres éducateurs, ce qui fit de son projet un savant mélange de tradition knoxienne, de philosophie des Lumières et de pédagogie contemporaine. De ces trois influences, il s'empara du meilleur de chacune pour ensuite en révéler toute la teneur. Il s'éclaira de la philosophie de Jean-Jacques Rousseau, de Helvétius, de William Godwin, de Jeremy Bentham et d'autres encore. Il s'initia aux pratiques et méthodes pédagogiques des célèbres Pestolazzi et Fallenberg. Il rencontra le Suisse Pestolazzi, lui-même fortement influencé par Rousseau. Il souhaitait éveiller l'intérêt de l'enfant en commençant par étudier l'aspect pratique d'un sujet ou d'un objet avant de s'intéresser à son contenu théorique. Il s'agit bien ici d'une approche empirique. Il faut favoriser une approche pratique, adaptée au milieu dans lequel évolue l'ouvrier. Le Suisse Fallenberg, quant à lui, disposait, entre autre, d'une École Normale. Convaincu par Fallenberg, Owen inscrivit ses propres fils dans cette école de pointe et d'une réputation hors pair. Après trois années d'études dans ce haut lieu de l'éducation, l'un de ses fils poursuivit l'œuvre paternelle.

À la même époque, l'évangélique Thomas Chalmers expérimentait des méthodes similaires dans ses paroisses urbaines de Glasgow. Il était également au fait des dernières méthodes pédagogiques. Toutefois, l'originalité de Owen résidait dans l'étroite corrélation entre pédagogie et industrie, entre instruction et formation et ce, au coeur de la manufacture.

me toute, le christianisme et la religion rationnelle ont en commun un même dessein d'éducation universelle : répandre des principes de vertu dans tous les foyers, et surtout répondre, dans l'immédiat, à la précarité sociale et morale des classes laborieuses. Owen aurait sans doute souhaité annihiler tout sens de christianisme dans sa fabrique mais au même titre qu'il jugeait voir du bon en l'Homme, il estimait aussi qu'il y avait du bon dans le christianisme. Dans son désir de révolution morale de la société, il s'était probablement heurté au pouvoir de la tradition presbytérienne, pouvoir qu'il avait suffisamment décrié. Ses associés Quakers avaient veillé au maintien de l'instruction religieuse dans la filature. Les ouvriers de New Lanark, dont la majorité avait connu l'école paroissiale et assistait au service du dimanche du temps de Dale, réclamaient également le maintien des pratiques liturgiques presbytériennes. La pression qu'incarnait la culture presbytérienne était donc forte et Owen, qui souhaitait maintenir la paix sociale au sein de sa communauté, acceptait, sans doute, de taire certains de ses principes rationnels en attendant des moments plus opportuns.

Afin de mettre en œuvre sa réforme sociale, il avait clairement stipulé, à la manière d'un prophète, que la première étape consistait à éliminer la religion traditionnelle et que la société était prête à vivre cette mutation. Mais force est de constater que la tradition était la plus forte. Il croyait néanmoins suffisamment à ses idées pour les mettre en application sous d'autres cieux, notamment à New Harmony, s'imaginant sans doute que le poids de la tradition serait moins lourd dans le Nouveau Monde.

Si l'objet du présent article n'était pas de traiter la notion de l'utopie stricto sensu, il convient cependant de conclure cette analyse sur les conséquences des rapports entre religion et rationalisme sur la notion d'utopie. C'est probablement le sens et la nature du terme communauté qui sont redéfinis à la lumière de ces rapports.

Afin de tenter de fournir quelques éléments de réponse, il convient de revenir un court instant sur la menace qu'incarne la religion traditionnelle pour son entreprise sociale. 
Owen voyait certainement dans la conversion à l'évangélisme un danger pour la société mais surtout une rivale de poids. Il redoutait - et il avait raison comme en témoigne un pasteur écossais en 1807 - que les ouvriers se convertissent à cette nouvelle expression de la foi, qui trouvait probablement sa source dans les Réveils de Cambuslang en 1742: "In Scotland weavers in Glasgow, Paisley, Perth, Dundee, etc., are among the first to join any new sect set up, the effect no doubt of their sedentary life and the melancholy monotony of their occupation. Where a great number of weavers are gathered, there new and gloomy notions of religion prevail ${ }^{25} »$.

Dans une certaine mesure, on ne peut pas faire abstraction du fait que la population, toutes classes confondues, ait perdu le sens de la communauté et de l'appartenance à un groupe : «Community sense collapsed in the industrial city $[. .].]^{26}$ ». Désormais, tout l'enjeu social est de remettre sur pied « la communauté » au sens large. Si ce dessein est partagé par le socialiste rationnel, Owen, et par les Églises, la finalité est somme toute différente. En effet, Owen souhaite rétablir ce sens à condition qu'il soit dépouillé de sa gangue dogmatique chrétienne. Finalement, Owen veut redonner tout son sens étymologique au mot religion, qui signifie relier et donc reconstruire la communauté. En fait, si la communauté est un microcosme de la société, c'est bel et bien la société, à plus grande échelle, qu'il s'agit aux yeux des réformateurs de reconstruire. Si, à la base, la communauté ne peut survivre aux aléas et aux pressions du contexte économique et social, alors l'entreprise communautaire est elle aussi vouée à l'échec et apparaît de facto utopique. Qu'il s'agisse d'une tentative de reconstruction religieuse ou rationnelle, la «communauté » telle qu'elle est pensée au $\mathrm{xIX}^{\mathrm{e}}$ siècle appartient, dans une certainement mesure, au monde de l'utopie.

\section{NOTES}

*. Abréviations : WRO (Works of Robert Owen) ; EW (Early Writings by Robert Owen) ; RSS (Religion and Society in Scotland by C.G. Brown).

1. Curtis S., J, History of Education in Great Britain, University Tutorial Press, London, 1948, p. 209.

2. Owen R., Evidence of the Select Committee on the State of Children Employed in Manufactories, 1816 ; in R.H. Campbell and J. B. Dow, Source Book of Scottish Economic and Social History, Oxford: Basil Blackwell, 1968, p. 153-55 in Civardi C., L'Écosse depuis 1528, Ophrys, 1998, p. 113.

3. Marx R., La Révolution industrielle en Grande Bretagne, Armand Colin, 1970, p. 177.

4. D'après Roland Marx, Owen aurait créé le mot socialiste en 1827 : «[...] le mot «socialiste » entre grâce à lui dans la langue anglaise, vers 1827 semble-t-il [...]», Marx R., La Révolution industrielle en Grande Bretagne, Armand Colin, 1970, p. 190.

5. Owen R., Works of Robert Owen : vol. 3, Book of the New Moral World: Seventh Part, p. 407. Friedrich Engels avait repris cette idée à son compte: «Robert Owen s'était approprié la doctrine des matérialistes du dix-huitième siècle : que le caractère de l'homme est le produit, d'un côté, de son organisation native, et, de l'autre, des circonstances qui l'environnent pendant sa vie et principalement pendant sa période de développement.» Engels, F., "Le matérialisme 
britannique", in Socialisme utopique et socialisme scientifique, Librairie de l'Humanité, Paris, 1924, trad. Paul Lafargue.

6. Après une succession d'enquêtes parlementaires, le parlement britannique a refusé de financer l'extension de la Kirk, devant autofinancer les constructions d'églises.

7. Hume D., Natural History of Religion (1757), Dialogues Concerning Natural Religion (1779) ; Locke J., Essay Concerning Human Understanding (1689).

8. Brown C.G., Religion and Society in Scotland since 1707, p. 108 (RSS en notes).

9. Owen R., Early Writings, vol. I, p. 227 (cité $E W$ dans le texte).

10. La Bible, 1 Corinthien $13,1-7,13$.

11. Dupuis S., La pensée et l'action d'un socialiste utopique, Robert Owen: 1771-1858., CNRS, Université de Toulouse, p. 321. Cet optimisme, parfois utopique, alimente suffisamment la volonté d'Owen pour qu'il poursuive ses projets.

12. Il y a ici une polémique entre catholiques et protestants qui trouve sa source dans l'interprétation des paroles des Apôtres Paul et Mathieu. Pour les uns, il s'agit du salut par les oeuvres et pour les autres par la grâce. Néanmoins, la Bible et le Premier Livre de Discipline appellent le croyant à faire acte de charité envers son prochain.

13. Chirpaz F., Raison et déraison de l'Utopie, l'Harmattan, 1999, p. 107.

14. Weber M., L'Éthique protestante et l'esprit du capitalisme (1904-1905), traduction par J. Chavy, Plon, 1964 ; nouvelle traduction par J.-P. Grossein, Gallimard 2003.

15. Smout T.C., A History of the Scottish People, 1560-1830, Collins/Fontana, 1969, p. 450.

16. Smollett T., The Expedition of Humphrey Clinker, 1771, Everyman, Londres, 1968, p. 221 in Civardi, C., L'Écosse depuis 1528, Ophrys, 1998, p. 83

17. McLaren D.J., David Dale, Robert Owen and the New Lanark Schools in the Scottish Educational Context, 1785-1824, p. 11.

18. Donnachie I.L. \& Hewitt G., Historic New Lanark: the Dale and Owen Community since 1785, Edinburgh University Press, 1994, p. 132.

19. Gordon P., Robert Owen, Perspectives: Revue trimestrielle d'éducation comparée (Paris, Unesco, Bureau International d'Education), vol. XXIV, $\mathrm{n}^{\circ}$ 1-2, 1994, p. 287-306.

20. Stone B.W., The Christian Messenger, le 25 décembre 1826, Georgetown, États-Unis, p. 44-46.

21. Chalmers T., The Influence of Bible Societies on the Necessities of the Poor, 1818.

22. De nombreux philanthropes s'essayaient comme Owen aux méthodes de Bell et de Lancaster. Il existait d'ailleurs des Lancasterian Schools à Glasgow, Édimbourg et Aberdeen qui fonctionnaient en dehors du circuit de l'école paroissiale.

23. Boyd W. \& King E., The History of Western Education, A \& C Black, London, 1977, p. 306-307.

24. Locke J., Some thoughts Concerning education and of the Conduct of the Understanding, Hacket Publishing Company, 1693, p. 34.

25. James Hall in Murray, D.B., "The social and religious origins of Scottish non-presbyterian Protestant dissent from 1730-1800", thèse de doctorat, Université de Saint Andrews, 1977, p. 214. RSS, p. 115.

26. Gilbert A.D., Religion and Society in Industrial England: Church, Chapel and Social Change 1740-1914, London, 1976, p. 113 in RSS, p. 9. 


\section{AUTEUR}

CLAIRE PUGLISI-KACZMAREK

Université de Provence 